Revista peruana de biología 26(3): 399 - 402 (2019) doi: http://dx.doi.org/10.15381/rpb.v26i3.15715 ISSN-L 1561-0837; eISSN: 1727-9933

Universidad Nacional Mayor de San Marcos

\section{Aportes sobre la dieta y distribución del Ocelote Leopardus pardalis (Linné 1758) en los altos Andes de Ecuador}

\section{Contributions on the diet and distribution of Ocelot Leopardus pardalis (Linné 1758) in the high Andes of Ecuador}

\author{
Carlos Nivelo-Villavicencio* ${ }^{*}$, Javier Fernández de Córdova ${ }^{1}$, Alis- \\ son Jiménez ${ }^{2}$, Pedro X. Astudillo ${ }^{3}$
}

Universidad del Azuay (MZUA), Cuenca, Ecuador. $\begin{array}{ll}\text { Presentado: } & 11 / 01 / 2019 \\ \text { Aceptado: } & 31 / 08 / 2019 \\ \text { Publicado online: } & 30 / 09 / 2019\end{array}$

Correspondencia:

Autor por correspondencia*

Carlos Nivelo-V.: cvillavicencio@uazuay.edu.ec Javier Fernández de C.: jfdcordova@gmail.com Alisson Jiménez: alissjimenez29@gmail.com Pedro X. Astudillo: pastudillow@uazuay.edu.ec

Otros datos de los autores / biografía:

1 Laboratorio de Vertebrados, Museo de Zoología. Av. 24 de mayo 7-77 y Hernán Malo, Teléfono: (593) 7 4091000, Fax: (593) 72815-997, Apartado 01.01.981, Cuenca, Ecuador.

2 Escuela de Biología, Ecología y Gestión. Facultad de Ciencia y Tecnología. Av. 24 de mayo 7-77 y Hernán Malo, Teléfono: (593) 74091000 , Fax: (593) 72815-997, Apartado 01.01.981, Cuenca, Ecuador. 3 Laboratorio de Ecología, Escuela de Biología, Ecología y Gestión. Av. 24 de mayo 7-77 y Hernán Malo, Teléfono: (593) 7 4091000, Fax: (593) 72815-997, Apartado 01.01.981, Cuenca, Ecuador.

ORCID Carlos Nivelo: 0000-0002-8502-3150 ORCID Javier Fernández: 0000-0003-3288-6822 ORCID Pedro X. Astudillo: 0000-0002-9945-9414

Citación:

Nivelo-Villavicencio C., J. Fernández de Córdova, A. Jiménez, P.X. Astudillo. 2019. Aportes sobre la dieta y distribución del Ocelote Leopardus pardalis (Linné 1758) en los altos Andes de Ecuador. Revista peruana de biología 26(3): 399 - 402 (Septiembre 2019). doi: http://dx.doi.org/10.15381/rpb.v26i3.15715

Palabras clave: bosque Montano; Cañar; Coendou rufescens; estribación de los Andes; valles interandinos.

Keywords: Andean foothills; Cañar; Coendou rufescens; inter-Andean valleys; Montane forest.

\section{Resumen}

El Ocelote, Leopardus pardalis es un carnívoro con amplia distribución en América del sur. En Ecuador, los registros se concentran en bosques tropicales de tierras bajas y en estribaciones de los Andes (<900 m de elevación), en donde disponen de una variedad de preseas, particularmente de pequeños mamíferos. Sin embargo, en las regiones alto-Andinas no se conoce sobre su presencia ni tampoco sobre su dieta. El 8 de noviembre de 2016 un macho adulto de L. pardalis que fue colectado muerto en la localidad de Usho, provincia del Cañar; el registro está localizado en la región alto-Andina al sur del Ecuador a 2818 m de elevación. Durante la evaluación del espécimen se encontraron varias púas digeridas y asociadas a la especie de Puerco espín de cola corta, Coendou rufescens. Este registro demuestra que L. pardalis ocurre al menos estacionalmente en la región alto-Andina del Ecuador; además demuestra la predación de especies alto-Andinas dentro de sus hábitos alimenticios.

\section{Abstract}

The Ocelot, Leopardus pardalis is a carnivore with wide distribution in South America. In Ecuador, most of the records come from lowland tropical forests and foothills of the Andes ( $<900 \mathrm{~m}$ at sea level), where this species has a variety of prey, particularly small mammals. However, in the high Andean regions, neither their occurrence nor their diet is known. On November 8, 2016 an adult male of L. pardalis was collected dead in the Usho locality, province of Cañar; the record is located in the high-Andean region in south of Ecuador at 2818 meters to sea level. During the evaluation of the specimen, several digested barbs were found and these were associated with the species of short-tailed porcupine, Coendou rufescens. This record shows that $L$. pardalis occurs at least seasonally in the high-Andean region of Ecuador; It also shows predation on the high-Andean species in their eating habits. 
El Ocelote, Leopardus pardalis (Felidae) está distribuido en América del Sur desde Venezuela hasta el norte de Argentina extendiéndose hacia Brasil Paraguay y Uruguay (Kitchener 1991, Murray \& Gardner 1997, Emmons \& Feer 1999). En el Ecuador, esta especie ocurre en regiones tropicales y subtropicales del occidente como así también de del oriente, llegando a ocupar estribaciones Andinas hasta los $1900 \mathrm{~m}$ de elevación (Tirira 2017). Este felino prefiere una variedad de hábitats boscosos, y, en particular bosques primarios y de sucesión secundaria (Tirira 2017); es de hábitos nocturnos, aunque puede presentar cierta actividad diurna (Konecny 1989, Sunquist 1992, Sunquist \& Sunquist 2002). La dieta consiste principalmente de pequeños mamíferos terrestres (Moreno et al. 2006); sin embargo, también incluye otros mamíferos medianos tales como ardillas (Sciuridae), zarigüeyas (Didelphidae), sajinos (Tayassuidae), cusumbos y cuchuchos (Procyonidae), y puercoespines del género Coendou (Bianchi 2007, Sánchez et al. 2008, Goldman 1920, De Villa Meza et al. 2002, Sunquist \& Sunquist 2009).

Buena parte de la información sobre la historia natural de L. pardalis ha sido generada mediante observaciones en regiones tropicales de tierras bajas y en estribaciones de los Andes (Bisbal 1986, De Villa Meza et al. 2002, Wang 2002). En consecuencia, información sobre localidades alto Andinas y, en particular, datos sobre la dieta en dichas localidades son poco conocidas (Murray \& Gardner, 1997). Por ejemplo, un solo estudio en los Andes de Colombia, reporta que L. pardalis se alimenta de roedores (Akodon bogotensis, Chilomys instans, Thomasomys aureus) y meso mamíferos como Didelphis albiventris, $\mathrm{Na}$ suella olivacea, Sylvilagus brasiliensis y Coendou rufescens en localidades con rangos de elevación entre $2100 \mathrm{~m}$ a $3800 \mathrm{~m}$ (Sánchez et al. 2008). Consecuentemente este trabajo pretende dar a conocer información importante recolectada en localidades alto Andinas y que aportará al conocimiento de la historia natural de L. pardalis.

El día 8 de noviembre de 2016 en la localidad de Usho, provincia de Cañar, un individuo macho adulto de L. pardalis fue encontrado muerto al costado de la vía Panamericana a 4 kilómetros de Biblián $\left(2^{\circ} 41^{\prime} \mathrm{S}, 78^{\circ} 53^{\prime} \mathrm{W}\right.$; $2818 \mathrm{~m}$; Fig. 1). La localidad de registro es un área rural dominada por pastos y cultivos, donde se pueden encontrar escasos remanentes de matorral montano que se encuentran bien dispersos a lo largo del paisaje; sin embargo, en zonas más apartadas y con pendientes pronunciadas (i.e. > 35\%) es posible encontrar fragmentos de bosque montano alto-Andino.
El ejemplar fue identificado como L. pardalis por medio de su morfometría externa y patrones de coloración que corresponden a las reportadas para esta especie (Murray \& Gardner 1997) (Tabla 1). El espécimen dorsalmente y en los flancos presenta manchas negras bien definidas, algunas se abren en forma de roseta y aparentan estar dispuestas a manera de líneas longitudinales negruzcas, en donde se puede apreciar un marrón pálido en su interior (Fig. 2), este patrón de coloración es distintivo en L. pardalis según Murray y Gardner (1997). El ejemplar fue procesado según el protocolo propuesto por Simmons y Muñoz-Saba (2005) y depositado en el Museo de Zoología de la Universidad del Azuay con el código (MZUA-MA261).

En el proceso de preparación del espécimen se pudo observar una herida abierta profunda en la parte anterior del muslo izquierdo y una fractura en el parietal y temporal izquierdo del cráneo; lo que sugiere que la causa de la muerte fue por atropellamiento. Los análisis del tracto digestivo evidenciaron la presencia de dos clases de púas localizadas en el estómago e intestino delgado. La primera clase de púas de 10 a $20 \mathrm{~mm}$ de longitud, presentan las puntas negras con la base anaranjada o amarillenta y fueron contabilizadas alrededor de 33 (Fig.

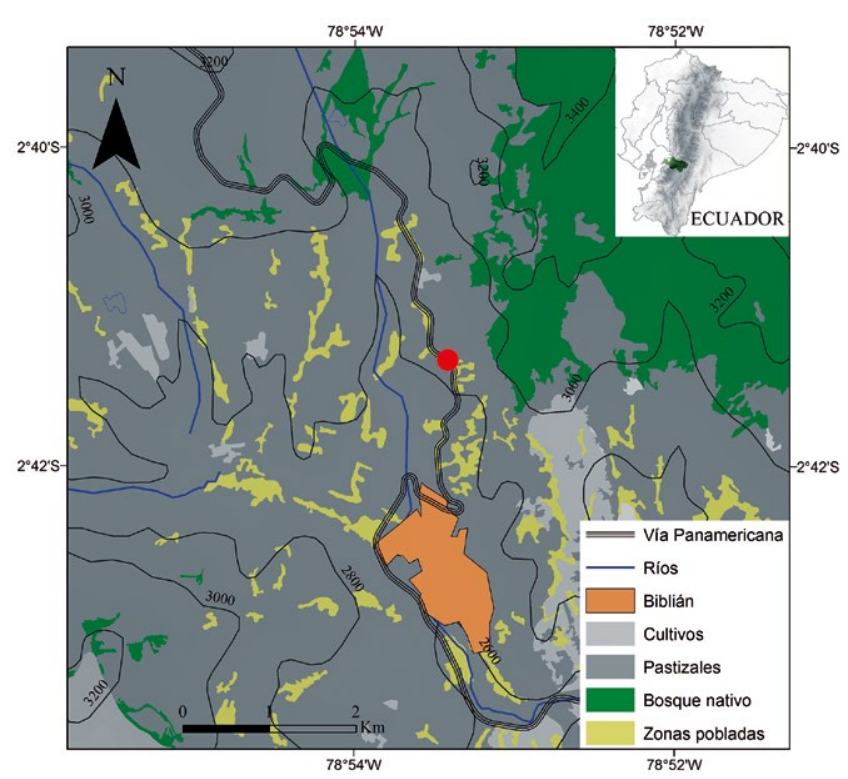

Figura 1. Nuevo registro de Leopardus pardalis (círculo rojo) en la localidad de Usho, provincia de Cañar, Ecuador.

Tabla 1. Medidas morfométricas $(\mathrm{mm})$ externas de un individuo macho adulto de Leopardus pardalis (MZUA-MA261), de la localidad del Usho, provincia del Cañar, Ecuador. Para la validación taxonómica se contrastó con medidas reportadas para machos por la literatura.

\begin{tabular}{lccc}
\hline Medidas & MZUA-MA261 & Tirira 2017 & Murray \& Gardner 1997 \\
\hline Largo total & 1230 & - & $1057-1230$ \\
Largo cabeza cuerpo & 830 & $700-1000$ & - \\
Largo de la cola & 400 & $255-410$ & $295-355$ \\
Largo de la pata posterior & 120 & $140-170$ & $142-151$ \\
Largo de la oreja & 70 & $50-65$ & - \\
\hline
\end{tabular}




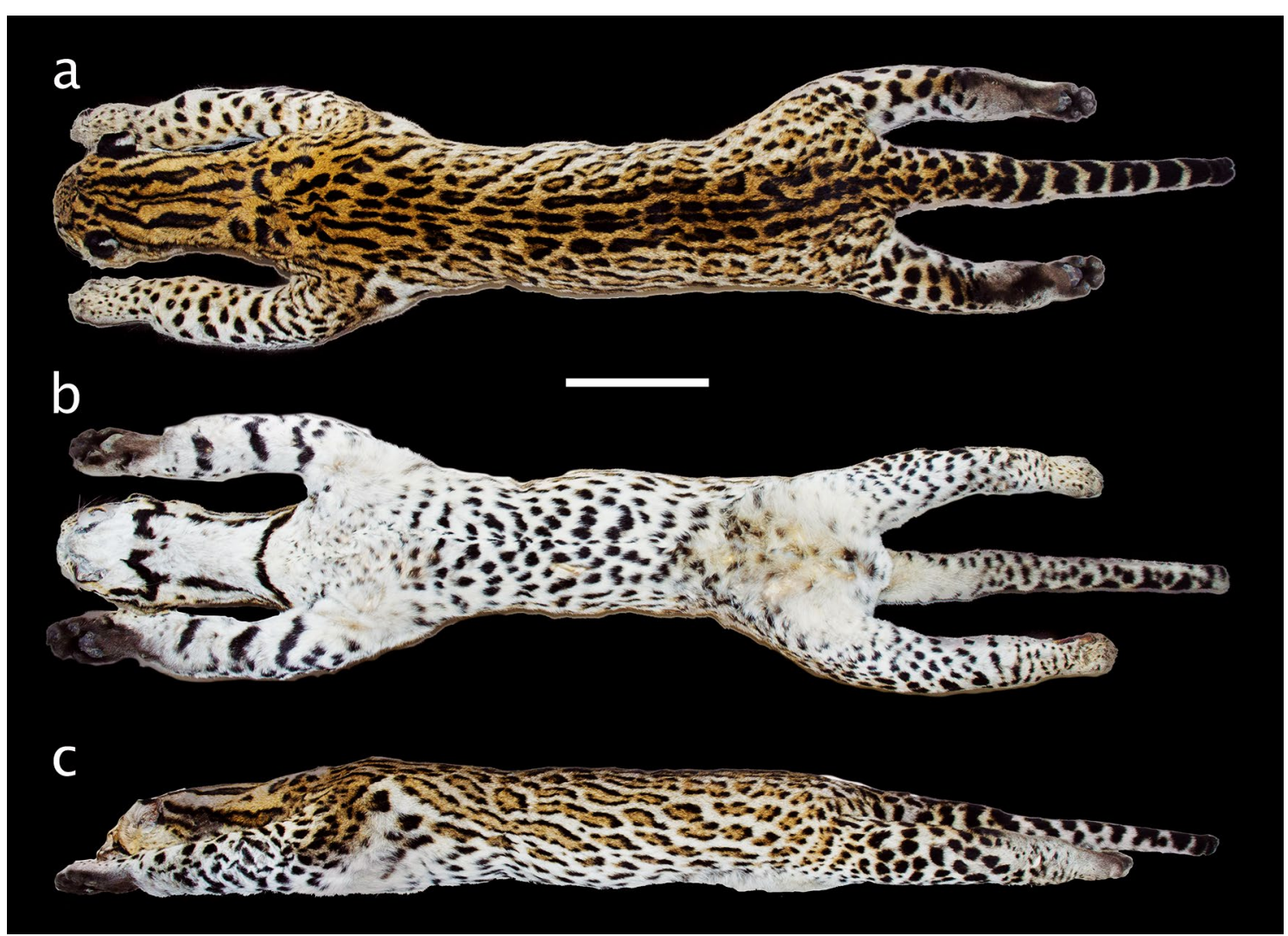

Figura 2. Espécimen macho adulto de Leopardus pardalis (MZUA-MA261) de la localidad de Usho, provincia de Cañar, Ecuador. a) vista dorsal, b) vista ventral y c) vista lateral. Barra $=15 \mathrm{~cm}$.

3a). Las púas de la segunda clase son más alargadas y delgadas $\sim 30 \mathrm{~mm}$, estas tienen las puntas rojizas con un negro brillante en el centro y con la base blanquecina, un total de 24 púas de este tipo fueron encontradas (Fig. 3b). Este hallazgo pone en evidencia la predación de puercoespines (Erethizontidae),sumado a esto, la localidad de registro de L. pardalis está dentro del rango de distribución de Coendou rufescens (Puerco espín de cola corta) y de acuerdo a los patrones de coloración descritos para las púas corresponderían a esta especie (Patton et al. 2015, Narváez-Romero et al. 2018), una de las es- pecies similares con la que podría confundirse es C. quichua, pero dentro de los patrones de coloración de esta especie, las púas no presentan coloración rojiza hacia la punta en ninguna parte del cuerpo, además de tener un rango de distribución por debajo de C. rufescens (Patton et al. 2015, Ramírez et al. 2016, Tirira 2017). Estas particularidades refuerzan la identificación de las púas y la predación de Coendou rufescens.

El presente registro reporta por primera vez la presencia de L. pardalis en los altos Andes de Ecuador, adi-

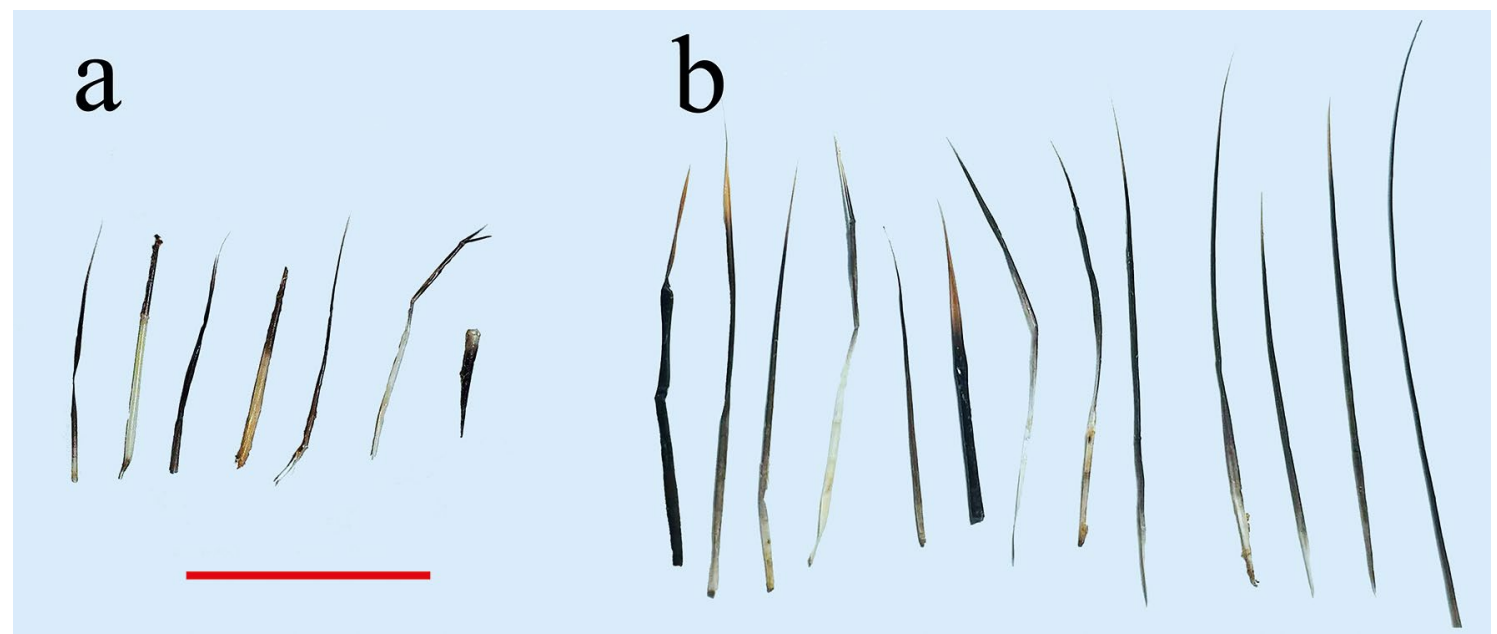

Figura 3. Púas de Coendou rufescens (Puerco espín de cola corta) encontradas en el tracto digestivo de Leopardus pardalis (Fig. 2), de la localidad Usho, provincia del Cañar, Ecuador: a) púas cortas sin coloración rojiza, b) púas largas con las puntas rojizas. Barra $=10 \mathrm{~mm}$. 
cionando una nueva localidad y extendiendo así el rango de ocurrencia geográfica de la especie hacia los valles interandinos del sur y, altitudinalmente en al menos 900 m s.n.m. En adición al nuevo registro, se aporta con información sobre la dieta; de tal modo, es posible inferir sobre sus hábitos alimenticios en regiones alto Andinas, pues especies del género Coendou ya han sido reportadas para la dieta de L. pardalis en otros sitios (Murray \& Gardner 1997, Sánchez et al. 2008). Dentro de este marco, este registro es evidencia de que L. pardalis puede ocupar hábitats alto Andinos y alimentarse de presas nativas de la región, al menos ocasionalmente.

\section{Literatura citada}

Bianchi R.D., S.L. Mendes. 2007. Ocelot (Leopardus pardalis) predation on primates in Caratinga Biological Station, southeast Brazil. Am J Primatol. 69 (10): 1173. Doi: https://doi.org/10.1002/ajp.20415

Bisbal E. 1986. Food habits of some neotropical carnivores in Venezuela (Mammalia, Carnivora). Mammalia, 50 (3): 329- 339. Doi: https://doi.org/10.1515/ mamm.1986.50.3.329

De Villa Meza A., E. Martinez Meyer \& C.A. López González. 2002. Ocelot (Leopardus pardalis) food habits in a tropical deciduous forest of Jalisco, Mexico. Am. Midl. Nat. 148 (1): 146-154. Doi: https://doi.org/10.1674/00030031(2002)148[0146:OLPFHI]2.0.CO;2

Emmons L.H. \& F. Feer. 1999. Mamíferos de los bosques húmedos de América Tropical, una guía de campo. 1era edición en español. 1era edición en español. Editorial FAN. Santa Cruz de la Sierra. 298 pp.

Goldman E.A. 1920. Mammals of Panama: (with Thirty-nine Plates), Smithsonian institution, 69 (2). Doi: https:// doi.org/10.5962/bhl.title.15684

Kitchener A. 1991. The natural history of the wild cats. Comstock Pub. Associates.

Konecny M. 1989. Movement patterns and food habits of four sympatric carnivore species in Belize, Central America. En: Redford, K.H.; Eisenberg, J.F., eds. Advances in Neotropical Mammalogy. The Sandhill Crane Press, Inc. (Gainesville, Fl.). p.243-264. 243า-264.

Moreno R.S., R.W. Kays \& JR. R. Samudio. 2006. Competitive release in diets of ocelot (Leopardus pardalis) and puma (Puma concolor) after jaguar (Panthera onca) decline. J. Mammal. 87 (4): 808-816. Doi: https://doi. org/10.1644/05-MAMM-A-360R2.1
Murray J.L \& G.L. Gardner. 1997. Leopardus pardalis. Mammalian species. 9 (548): 1-10. https://doi. org $/ 10.2307 / 3504082$

Narváez-Romero C., C. Reyes-Puig, D. Valle, et al. 2018. New records and estimation of the potential distribution of the stump-tailed porcupine Coendou rufescens. Therya. 9 (2): 137. https://doi.org/10.12933/therya-18-581

Patton J.L., U.F. Pardiñas \& G. D'Elía. 2015. Mammals of South America, volume 2. University of Chicago Press. 1336 pp. https://doi.org/10.7208/chicago/9780226169606.001.0001

Sánchez F., B. Gómez-Valencia, S.J. Álvarez, et al. 2008. First data on the food habits of the Ocelot, Leopardus pardalis, in a Colombian Andean forest. Revista UDCA Actualidad y Divulgación Científica. 11 (2): 101-107.

Simmons J. E. \& Y. Muñoz-Saba. 2005. Cuidado, manejo y conservación de las colecciones biológicas. Universidad Nacional de Colombia. Conservation International. $288 \mathrm{pp}$.

Sunquist M. 1992. The ecology of the ocelot: the importance of incorporating life history traits into conservation plans. In Memorias del Simposio organizado por Fudeci-Caracas. Vol. 1991 117-128.

Sunquist M.E. \& F.C. Sunquist. 2002. Wild cats of the World. Chicago: The University of Chicago Press. 262 pp. https:// doi.org/10.7208/chicago/9780226518237.001.0001

Sunquist ME \& F.C. Sunquist. 2009. Family Felidae (cats) In: Wilson D.E \& R.A. Mittermeier (ed). Handbook of the Mammals of the World. Vol. 1. Carnivores. Barcelona: Lynx Editions. 54-169.

Tirira D.G. 2017. Mamíferos del Ecuador. Guía de campo. Ediciones Murciélago Blanco. Publicación Especial de los Mamíferos del Ecuador 10. Quito. 576 pp.

Wang E. 2002. Diets of ocelots (Leopardus pardalis), margays (L. wiedii), and oncillas (L. tigrinus) in the Atlantic rainforest in southeast Brazil. Stud. Neotrop. Fauna Environ.37 (3): 207-212. https://doi.org/10.1076/ snfe.37.3.207.8564

\section{Agradecimientos:}

Agradecemos a Jacinto Guillén de la Universidad del Azuay por su apoyo constante a nuestras investigaciones. Juan Carlos Sánchez y Amanda Quezada colaboraron activamente en la preparación del ejemplar.

Conflicto de intereses:

Los autores no incurren en conflictos de intereses.

Rol de los autores:

CN-V, JFC, PXA prepararon el manuscrito y revisaron el contenido del mismo. Las fotografías de los especímenes fueron preparadas por $\mathrm{CN}-\mathrm{V}$.

Fuentes de financiamiento:

Los autores manifiestan no haber contado con un financiamiento específico.

Aspectos éticos / legales:

El Museo de Zoología de la Universidad del Azuay cuenta con la patente ambiental FAUS-UDA-MUSEO-DE ZOOLOGÍA-003-2018. 